\title{
Evidence-based lead mitigation strategies: Are the DIY lead mitigation recommendations by health departments supported by research?
}

\author{
Seth Losiewicz ${ }^{1}$, Heidi Beidinger-Burnett ${ }^{2}$, Christopher Knaub² \\ ${ }^{1}$ Indiana University School of Medicine, Indiana Clinical and Translational Science Institute, \\ 2University of Notre Dame, Eck Institute for Global Health
}

Background: Legislation outlawing leaded paint in 1978 and tetraethyl lead from gasoline in 1992 effectively decreased blood lead levels (BLLs), but the effects of lead are still felt in the United States to this day. There is no safe level of lead in the body, and even low level lead exposure can lead to cognitive and developmental delays such as learning delay and disabilities; low IQ; and attention-deficit/hyperactivity disorder. The CDC has determined that a $\mathrm{BLL}$ of $5 \mu \mathrm{g} / \mathrm{dL}$ or higher is cause for environmental and educational intervention. Today, the EPA estimates that there are approximately 24 million housing units containing significant lead hazards, with 4 million being home to children. Many of the families that are faced with lead hazards in their homes are minorities and low socioeconomic status. To assist families with possible lead hazards in the home, interim controls of lead hazards may be paramount to reducing lead levels in the household in a simple, cost effective manner.

Methods: To that end, our research had two aims (a) conduct a systematic literature review to learn about do-it-yourself (DIY) interim controls that are evidence-based and effective in reducing lead hazards in the home and (b) conduct interviews with key personnel at each state health department to learn more about their lead prevention programs and the DIY mitigation strategies recommended to families

Results: Our provisional findings suggest there is an inconsistent, wide range of mitigation strategies recommended by the state health departments that often lack empirical evidence. . While our literature review identified effective interim controls, the literature is outdated.

Conclusion and Potential Impact: We recommend more studies are needed to identify cost effective interim controls and the standardization of health department DIY interim controls across the nation. 